\title{
Bergman kernel and oscillation theory of plurisubharmonic functions
}

\author{
Bo-Yong Chen ${ }^{1} \cdot \mathrm{Xu}$ Wang $^{2}$
}

Received: 8 September 2019 / Accepted: 7 June 2020 / Published online: 30 June 2020

(c) The Author(s) 2020

\begin{abstract}
Based on Harnack's inequality and convex analysis we show that each plurisubharmonic function is locally BUO (bounded upper oscillation) with respect to polydiscs of finite type but not for arbitrary polydiscs. We also show that each function in the Lelong class is globally BUO with respect to all polydiscs. A dimension-free BUO estimate is obtained for the logarithm of the modulus of a complex polynomial. As an application we obtain an approximation formula for the Bergman kernel that preserves all directional Lelong numbers. For smooth plurisubharmonic functions we derive a new asymptotic identity for the Bergman kernel from Berndtsson's complex Brunn-Minkowski theory, which also yields a slightly better version of the sharp Ohsawa-Takegoshi extension theorem in some special cases.
\end{abstract}

Keywords BUO · Plurisubharmonic function - Bergman kernel · Remez inequality · Directional Lelong number · Complex Brunn-Minkowski theory · Ohsawa-Takegoshi theorem

Mathematics Subject Classification 32A25 · 53C55

\section{Introduction}

Let $\Omega$ be a domain in $\mathbb{C}^{n}$ and $P S H(\Omega)$ the set of plurisubharmonic (psh) functions on $\Omega$. Recall that each $\phi \in P S H(\Omega)$ satisfies the following mean-value inequality:

$$
\phi(z) \leq \frac{1}{|S|} \int_{S} \phi=: \phi_{S}
$$

Bo-Yong Chen is supported by NSF Grant 11771089 and Gaofeng grant from School of Mathematical Sciences, Fudan University.

\footnotetext{
$\triangle \quad$ Xu Wang

xu.wang@ntnu.no

Bo-Yong Chen

boychen@fudan.edu.cn

1 Department of Mathematical Sciences, Fudan University, Shanghai 20043, China

2 Department of Mathematical Sciences, Norwegian University of Science and Technology, 7491 Trondheim, Norway
} 
whenever $S$ is a ball or a polydisc, with center $z$. Here $|S|$ denotes the Lebesgue measure of $S$ and $\int_{S}$ means the Lebesgue integral. The above inequality implies $\phi \in L_{\text {loc }}^{1}(\Omega)$ and suggests to estimate the difference $\left|\phi-\phi_{S}\right|$. The concept of BMO functions then enters naturally. Let $\mathcal{S}=\mathcal{S}(\Omega)$ be a family of relatively compact open subsets in $\Omega$. We say that $\phi \in L_{\text {loc }}^{1}(\Omega)$ has bounded mean oscillation (BMO) with respect to $\mathcal{S}$ if

$$
\sup _{S \in \mathcal{S}} M O_{S}(\phi)<\infty, \quad M O_{S}(\phi):=\frac{1}{|S|} \int_{S}\left|\phi-\phi_{S}\right| .
$$

Let $B M O(\Omega, \mathcal{S})$ denote the set of functions which are BMO with respect to $\mathcal{S}$. When $\mathcal{S}$ is the set of balls in $\Omega$, this is the original definition of BMO functions due to John-Nirenberg [13]. A classical example of BMO functions is $\log |z|$. It is also convenient to introduce local BMO functions as follows. For an open set $\Omega_{0} \subset \subset \Omega$ we define $\left.\mathcal{S}\right|_{\Omega_{0}}$ to be the sets of all $S \in \mathcal{S}$ which are relatively compact in $\Omega_{0}$. Let $B M O_{\text {loc }}(\Omega, \mathcal{S})$ be the set of functions on $\Omega$ which belong to $B M O\left(\Omega_{0},\left.\mathcal{S}\right|_{\Omega_{0}}\right)$ for every open set $\Omega_{0} \subset \subset \Omega$.

By using pluripotential theory, Brudnyi [6] was able to show that each psh function is locally BMO with respect to balls (see also [7] for stronger results concerning subharmonic functions in the plane). Recently, the first author found another approach to local BMO properties of psh functions by using the Riesz decomposition theorem and some basic facts of psh functions (cf. [9]). Benelkourchi et al. [1] showed that every function in the Lelong class $\mathcal{L}$ is globally BMO with respect to balls. Recall that

$$
\mathcal{L}=\left\{u \in \operatorname{PSH}\left(\mathbb{C}^{n}\right): \lim \sup _{|z| \rightarrow \infty}(u(z)-\log |z|)<\infty\right\} .
$$

In this paper we propose a new and simpler approach based on the following basic observation:

It is easier to look at the upper oscillation instead of the mean oscillation for psh functions.

To define the upper oscillation one simply uses $\sup _{S} \phi$ instead of $\phi_{S}$ :

$$
U O_{S}(\phi):=\frac{1}{|S|} \int_{S}\left|\phi-\sup _{S} \phi\right|=\sup _{S} \phi-\phi_{S} .
$$

Note that $-U O_{S}(-\phi)$ is exactly the lower oscillation introduced by Coiffman-Rochberg (cf. [10], see also [16] for further properties). Since

$$
M O_{S}(\phi)=\frac{1}{|S|} \int_{S}\left|\phi-\phi_{S}\right|=\frac{2}{|S|} \int_{\phi<\phi_{S}}\left(\phi_{S}-\phi\right) \leq 2 U O_{S}(\phi),
$$

we see that bounded upper oscillation (BUO) implies BMO. One may define $B U O(\Omega, \mathcal{S}$ ) and $B U O_{\text {loc }}(\Omega, \mathcal{S})$ analogously as the case of BMO.

Let $\mathcal{P}=\mathcal{P}(\Omega)$ denote the set of relatively compact polydiscs in $\Omega$ and $\mathcal{P}_{N}$ the set of polydiscs $P \subset \subset \Omega$ of finite type $N$, i.e.,

$$
\max \left\{r_{j}\right\} \leq \min \left\{r_{j}^{1 / N}\right\},
$$

where $N>0$ and $\left\{r_{j}\right\}_{1 \leq j \leq n}$ is the polyradius of $P$.

Based on Harnack's inequality and convex analysis, we are able to show the following

Theorem 1.1 (1) $P S H(\Omega) \subset B U O_{\mathrm{loc}}\left(\Omega, \mathcal{P}_{N}\right) \subset B M O_{\mathrm{loc}}\left(\Omega, \mathcal{P}_{N}\right)$;

(2) $P S H\left(\mathbb{D}^{n}\right) \nsubseteq B M O_{\text {loc }}\left(\mathbb{D}^{n}, \mathcal{P}\right)$ for $n \geq 2$, where $\mathbb{D}^{n}$ is the unit polydisc;

(3) $\mathcal{L} \subset B U O\left(\mathbb{C}^{n}, \mathcal{P}\right)$; more precisely, for every $\phi \in P S H\left(\mathbb{C}^{n}\right)$ with

$$
\phi\left(z_{1}, \ldots, z_{n}\right) \leq c+\max _{1 \leq j \leq n} \log \left(1+\left|z_{j}\right|\right), \quad \forall\left(z_{1}, \ldots, z_{n}\right) \in \mathbb{C}^{n},
$$

where $c$ is a constant, we have $U O_{P}(\phi)<3^{n}$ for all polydiscs $P$ in $\mathbb{C}^{n}$. 
For $(\operatorname{deg} p)^{-1} \log |p| \in \mathcal{L}$ where $p$ is a complex polynomial, we even obtain a dimensionfree BUO estimate with respect to all compact convex sets.

Theorem 1.2 For every non-empty compact convex set $A$ in $\mathbb{C}^{n}$, we have

$$
U O_{A}(\log |p|) \leq \gamma \cdot \operatorname{deg} p,
$$

for all $p \in \mathbb{C}\left[z_{1}, \ldots, z_{n}\right]$. Here the constant $\gamma \in(1,2)$ is determined by

$$
\gamma+\log (\gamma-1)=0 .
$$

Remark (i) The above estimate is sharp, in fact, there exists a line segment $A$ in $\mathbb{C}$ such that

$$
U O_{A}(\log |z|)=\gamma .
$$

(ii) In particular, if $A$ is a compact convex set in $\mathbb{R}^{n} \subset \mathbb{C}^{n}$ and all coefficients of $p$ are real, then we have

$$
U O_{A}(\log |p|) \leq \gamma \cdot \operatorname{deg} p<2 \operatorname{deg} p,
$$

which is closely related the classical Remez inequality for real polynomials. Theorem 1.2 also suggests to study the Remez inequality for complex polynomials (see [1] and [8] for related results).

(iii) Notice that $1.278<\gamma<1.279$. By (1.2) we have

$$
M O_{A}(\log |p|) \leq 2 \gamma \cdot \operatorname{deg} p<2.558 \cdot \operatorname{deg} p .
$$

Such dimension-free estimate (with a slightly better constant $2+\log 2 \approx 2.301$ ) was first obtained by Nazarov et al. [15]. Our proof of Theorem 1.2 is elementary, however.

For $\phi \in P S H(\Omega)$ we define the (weighted) Bergman kernel by

$$
K_{\phi, \Omega}(z)=\sup \left\{|f(z)|^{2}: f \in \mathcal{O}(\Omega), \int_{\Omega}|f|^{2} e^{-\phi} \leq 1\right\} .
$$

For a vector $a=\left(a_{1}, \ldots, a_{n}\right)$ with all $a_{j}>0$ we set

$$
P_{r^{a}}:=\left\{z \in \mathbb{C}^{n}:\left|z_{j}\right| \leq r^{a_{j}}, 1 \leq j \leq n\right\} .
$$

It was shown in [9] that if $\phi$ is psh on the closure of the unit ball $\mathbb{B}^{n}$ and $a_{0}=(1,1 / 2, \ldots, 1 / 2)$ then

$$
\lim _{r \rightarrow 0+} \frac{\log K_{\varepsilon \phi, \mathbb{B}^{n}}(1-r, 0, \ldots, 0)}{\log 1 / r}=n+1-\varepsilon \cdot \lim _{r \rightarrow 0+} \frac{\sup _{z \in P_{r} a_{0}} \phi(1+z)}{\log r}
$$

provided $\varepsilon \ll 1$, where $1+z=\left(1+z_{1}, z_{2}, \ldots, z_{n}\right)$. The limit in RHS of the above inequality is called the $a_{0}$-directional Lelong number of $\phi$ at $(1,0, \ldots, 0)$ (see [14]).

Here we will present an analogous but independent result, as an application of Theorem 1.1. For $\phi \in P S H\left(\mathbb{D}^{n}\right)$ and $t \in \mathbb{D}^{n}$ we define

$$
\phi^{t}(z):=\phi(t z), \quad t z:=\left(t_{1} z_{1}, \ldots, t_{n} z_{n}\right) .
$$

A fundamental result of Berndtsson [2] implies that

$$
F(\phi):(t, z) \mapsto \log K_{\phi^{t}, \mathbb{D}^{n}}(z)
$$

is psh on $\mathbb{D}^{n} \times \mathbb{D}^{n}$. 
Theorem 1.3 For each $a=\left(a_{1}, \ldots, a_{n}\right)$ with all $a_{j}>0$, there exists a number $\varepsilon_{0}=$ $\varepsilon_{0}(a, \phi, \Omega)$ such that

$$
\lim _{r \rightarrow 0+} \frac{\sup _{t \in P_{r} a} F(\varepsilon \phi)(t, 0) / \varepsilon}{\log r}=\lim _{r \rightarrow 0+} \frac{\sup _{z \in P_{r} a} \phi(z)}{\log r}
$$

holds for all $\varepsilon \leq \varepsilon_{0}$.

Although Theorem 1.3 makes sense only when $\phi$ is singular at the origin, it is of independent interest to study the relation between $F(\phi)$ and $\phi$ for smooth $\phi$.

Theorem 1.4 Let $\phi$ be a smooth psh function on $\mathbb{D}^{n}$. Then

$$
\lim _{t \rightarrow 0} \frac{\partial^{2} F(\phi)}{\partial t_{j} \partial \bar{t}_{k}}(t, 0)=\left\{\begin{array}{l}
\frac{1}{2} \cdot \frac{\partial^{2} \phi}{\partial z_{j} \partial \bar{z}_{j}}(0), \quad \text { if } j=k ; \\
0, \quad \text { if } j \neq k .
\end{array}\right.
$$

In particular $F(\phi)(t, 0)$ is strictly psh at $t=0$ if $\phi$ is strictly psh at $z=0$.

Remark Since $F(\phi)(t, 0)$ depends only on $\left(\left|t_{1}\right|, \ldots,\left|t_{n}\right|\right)$, it follows from the psh property of $F(\phi)$ that

$$
\log \frac{e^{\phi(0)}}{\pi^{n}}=F(\phi)(0,0) \leq F(\phi)(t, 0)=\log K_{\phi^{t}, \mathbb{D}^{n}}(0) .
$$

Letting $t$ tend to $(1, \ldots, 1)$, we obtain the sharp Ohsawa-Takegoshi estimate (cf. [5]; see also $[4,12])$ :

$$
K_{\phi, \mathbb{D}^{n}}(0) \geq \frac{e^{\phi(0)}}{\pi^{n}} .
$$

Theorem 1.4 suggests that one should have a better lower bound for $K_{\phi}, \mathbb{D}^{n}$ in case $\phi$ is strictly psh.

\section{An enlightening example}

To explain why BUO is easier than BMO, we will show that the upper oscillation of $\log |z|$ with respect to discs is computable. Recall that

$$
U O_{B}(\log |z|):=\sup _{B} \log |z|-(\log |z|)_{B}
$$

for every $\operatorname{disc} B$ in $\mathbb{C}$.

Lemma 2.0.1 Fix $\hat{z} \in \mathbb{C}$ and set

$$
I(c):=\frac{1}{2 \pi} \int_{0}^{2 \pi} \log \left|\hat{z}+c e^{i \theta}\right| d \theta, \quad c>0 .
$$

Then we have

$$
I(c)= \begin{cases}\log |\hat{z}| & \text { if } c \leq|\hat{z}| \\ \log c & \text { if } c>|\hat{z}| .\end{cases}
$$


Proof If $c \leq|\hat{z}|$ then $\log |z|$ is harmonic in the disc $\{z:|z-\hat{z}|<c\}$, so that $I(c)=\log |\hat{z}|$, in view of the mean-value equality. For $c>|\hat{z}|$ we may write

$$
I(c)=\frac{1}{2 \pi} \int_{0}^{2 \pi} \log \left|\hat{z} e^{i \theta}+c\right| d \theta .
$$

As $\log |z|$ is harmonic in $\{z:|z-c|<|\hat{z}|\}$, we get $I(c)=\log c$.

Proposition 2.0.1 For any disc $B$ we have

$$
U O_{B}(\log |z|) \leq \log \frac{\sqrt{5}+1}{2}+\frac{\sqrt{5}-1}{4} .
$$

Moreover, the bound is sharp.

Proof Suppose $B=\{z:|z-\hat{z}|<b\}$. By Lemma 2.0.1 we have

$$
(\log |z|)_{B}=\log |\hat{z}|, \quad \text { if } b \leq|\hat{z}|,
$$

and if $b>|\hat{z}|$ then

$$
\begin{aligned}
(\log |z|)_{B} & =\frac{1}{\pi b^{2}} \int_{0}^{b} 2 \pi c \cdot I(c) d c \\
& =\log b-\frac{1}{2}\left(1-\frac{|\hat{z}|^{2}}{b^{2}}\right) .
\end{aligned}
$$

It follows that

$$
U O_{B}(\log |z|)= \begin{cases}\log (b+|\hat{z}|)-\log |\hat{z}| & \text { if } b \leq|\hat{z}| \\ \log (b+|\hat{z}|)-\log b+\frac{1}{2}\left(1-\frac{|\hat{z}|^{2}}{b^{2}}\right) & \text { if } b>|\hat{z}|\end{cases}
$$

If $b \leq|\hat{z}|$ then

$$
U O_{B}(\log |z|)=\log \left(\frac{b}{|\hat{z}|}+1\right) \leq \log 2 .
$$

For $b>|\hat{z}|$ we set $x=|\hat{z}| / b$ and write $U O_{B}(\log |z|)$ as

$$
f(x)=\log (1+x)+\frac{1}{2} \cdot\left(1-x^{2}\right), \quad 0<x<1 .
$$

Since

$$
f^{\prime}(x)=\frac{1}{1+x}-x,
$$

we see that $f$ is increasing on $[0, \hat{x}]$ and decreasing on $[\hat{x}, 1]$, where $\hat{x}=\frac{\sqrt{5}-1}{2}$. Notice that

$$
f(\hat{x})=\log \frac{\sqrt{5}+1}{2}+\frac{\sqrt{5}-1}{4} \text {. }
$$

Thus

$$
U O_{B}(\log |z|) \leq \log \frac{\sqrt{5}+1}{2}+\frac{\sqrt{5}-1}{4}
$$

and the equality holds if and only if

$$
\frac{|\hat{z}|}{b}=\frac{\sqrt{5}-1}{2} .
$$

This finishes the proof. 


\section{Proof of Theorem 1.1}

\subsection{One dimensional case}

Let $\Omega$ be a domain in $\mathbb{C}$ and $\phi$ a subharmonic function on $\Omega$. Recall that

$$
U O_{B}(\phi)=\sup _{B} \phi-\phi_{B}
$$

where $B=\{z:|z-\hat{z}|<r\} \subset \Omega$. The idea is to use Harnack's inequality and a convexity lemma. Let us write

$$
U O_{B}(\phi)=I_{1}+I_{2}
$$

where

$$
I_{1}=\sup _{B} \phi-\phi_{\partial B}, \quad I_{2}:=\phi_{\partial B}-\phi_{B},
$$

with $\phi_{\partial B}$ being the mean-value of $\phi$ over the boundary $\partial B$. For each $\tau>0$ we set

$$
\tau B=\{z:|z-\hat{z}|<\tau r\} .
$$

Applying Harnack's inequality to the nonpositive subharmonic function $\psi:=\phi-\sup _{B} \phi$, we get

$$
\sup _{\frac{1}{2} B} \psi=\sup _{\partial\left(\frac{1}{2} B\right)} \psi \leq \frac{1}{3} \cdot \psi_{\partial B}
$$

i.e.,

$$
I_{1} \leq 3\left(\sup _{B} \phi-\sup _{\frac{1}{2} B} \phi\right) .
$$

Here the constant $1 / 3$ comes from the Poisson kernel of the unit disc since

$$
\inf _{|z|=1 / 2} \frac{1-|z|^{2}}{|1-z|^{2}}=\frac{1}{3} .
$$

The following fact explains why we need such an estimate.

Fact $1 J_{1}:=\sup _{B} \phi-\sup _{\frac{1}{2} B} \phi$ is continuous in $\hat{z}$ and $r$ respectively; moreover, it is increasing with respect to $r$.

Proof $\operatorname{Since} \sup _{B} \phi$ is a convex function of $\log r$ (see [11, Corollary 5.14]), it follows that $J_{1}$ is a continuous increasing function of $r$. The continuity of $J_{1}$ in $\hat{z}$ is obvious.

Let $\Omega_{0}$ be a relatively compact open subset in $\Omega$. Let $\delta_{0}$ denote the distance between $\overline{\Omega_{0}}$ and $\partial \Omega$. By the above fact we see that if the radius $r$ of $B \subset \Omega_{0}$ is less than $\delta_{0} / 2$ then

$$
I_{1} \leq 3 \sup _{\hat{z} \in \Omega_{0}} J_{1}\left(\hat{z}, \delta_{0} / 2\right)<\infty
$$

and if $r \geq \delta_{0} / 2$ then

$$
I_{1} \leq 3 \sup _{\Omega_{0}} \phi-3 \inf _{\hat{z} \in \Omega_{0}} \sup _{\left\{|z-\hat{z}|<\delta_{0} / 4\right\}} \phi<\infty .
$$

To estimate $I_{2}$, we need the following convexity lemma which was communicated to the second author by Bo Berndtsson: 
Lemma 3.0.2 Let $d \mu$ be a probability measure on a Borel measurable subset $S$ in $\mathbb{R}^{n}$ with barycenter $\hat{t} \in \mathbb{R}^{n}$. Let $f$ be a convex function on $\mathbb{R}^{n}$. Then

$$
\int_{S} f d \mu \geq f(\hat{t}) .
$$

Proof Since $f$ is convex, there exists an affine function $l$ such that $f(\hat{t})=l(\hat{t})$ and $f \geq l$ on $\mathbb{R}^{n}$, which implies

$$
\int_{S} f d \mu \geq \int_{S} l d \mu=l(\hat{t})=f(\hat{t}),
$$

where the first equality follows from the definition of barycenter.

With $f(t):=\phi_{\left\{z:|z-\hat{z}|=e^{t} r\right\}}$ we have

$$
\begin{aligned}
I_{2} & =f(0)-\frac{1}{\pi r^{2}} \int_{-\infty}^{0} 2 \pi e^{t} r \cdot f(t) d\left(e^{t} r\right) \\
& =f(0)-\int_{-\infty}^{0} f(t) d\left(e^{2 t}\right) .
\end{aligned}
$$

Since $f(t)$ is convex and $d\left(e^{2 t}\right)$ is a probability measure on $(-\infty, 0)$ with barycenter at $t=-1 / 2$, it follows from Lemma 3.0.2 that

$$
\int_{-\infty}^{0} f(t) d\left(e^{2 t}\right) \geq f(-1 / 2),
$$

which implies

$$
I_{2} \leq J_{2}:=f(0)-f(-1 / 2) .
$$

Since $f$ is convex, we get an analogous conclusion as Fact 1:

Fact $2 J_{2}$ is continuous in $\hat{z}$ and $r$ respectively; moreover, it is increasing with respect to $r$.

By a similar argument as above, we may verify that

$$
\sup _{B \subset \Omega_{0}} I_{2}<\infty \text {. }
$$

\subsection{High dimensional case}

The following result plays the role of Fact 1, 2 .

Lemma 3.0.3 Let $g(t)=g\left(t_{1}, \ldots, t_{n}\right)$ be a convex function on $(-\infty, 2)^{n}$ which is increasing in each variable. Then

$$
\sup _{t \in A_{N}}[g(t)-g(t-1)] \leq n N[g(1, \ldots, 1)-g(0)],
$$

where $t-1:=\left(t_{1}-1, \ldots, t_{n}-1\right), N \geq 1$ and

$$
A_{N}:=\left\{t \in(-\infty, 0]^{n}: \max \left\{-t_{j}\right\} \leq N \min \left\{-t_{j}\right\}\right\} .
$$

Proof A standard regularization process reduces to the case when $g$ is smooth. Set

$$
f(a)=g\left(t_{1}+a, \ldots, t_{n}+a\right):=g(t+a) .
$$


We have

$$
f(0)-f(-1)=\int_{-1}^{0} f^{\prime}(a) d a=\int_{-1}^{0} \sum g_{j}(t+a) d a
$$

where $g_{j}:=\frac{\partial g}{\partial t_{j}}$. Notice that

$$
\sum g_{j}(t+a) \leq \frac{1}{\min \left\{-t_{j}-a\right\}} \sum\left(-t_{j}-a\right) g_{j}(t+a)
$$

and

$$
\sum\left(-t_{j}-a\right) g_{j}(-s(t+a))=\frac{d g(-s(t+a))}{d s}
$$

is an increasing function of $s \in(-\infty, 0)$ by convexity of $g$. Thus we have

$$
\sum\left(-t_{j}-a\right) g_{j}(t+a) \leq \sum\left(-t_{j}-a\right) g_{j}(0) \leq \max \left\{-t_{j}-a\right\} \sum g_{j}(0),
$$

which implies

$$
\sum g_{j}(t+a) \leq \frac{\max \left\{-t_{j}-a\right\}}{\min \left\{-t_{j}-a\right\}} \sum g_{j}(0) .
$$

For any $t \in A_{N}$, we have $t+a \in A_{N}$ (since $a \leq 0$ ), so that

$$
\frac{\max \left\{-t_{j}-a\right\}}{\min \left\{-t_{j}-a\right\}} \leq N \text {. }
$$

Thus

$$
g(t)-g(t-1) \leq N \sum g_{j}(0) .
$$

Since $g$ is convex and increasing, we have

$$
g_{j}(0) \leq g(1, \ldots, 1)-g(0),
$$

which finishes the proof.

Let

$$
P:=\left\{z \in \mathbb{C}^{n}:\left|z_{j}-\hat{z}_{j}\right|<r_{j}, 1 \leq j \leq n\right\} \subset \Omega
$$

be a polydisc of type $N$, i.e.,

$$
\max \left\{r_{j}\right\} \leq \min \left\{r_{j}^{1 / N}\right\}
$$

Similar as above, we write

$$
U O_{P}(\phi)=\sup _{P} \phi-\phi_{P}=I_{1}+I_{2},
$$

where

$$
I_{1}:=\sup _{P} \phi-\phi_{\partial P}, \quad I_{2}:=\phi_{\partial P}-\phi_{P}
$$

and

$$
\partial P:=\left\{z \in \mathbb{C}^{n}:\left|z_{j}-\hat{z}_{j}\right|=r_{j}, 1 \leq j \leq n\right\}
$$

is the Shilov boundary of $P$. Applying Harnack's inequality (see [14, p. 186]) $n$-times, we get the following 
Lemma 3.0.4 $I_{1} \leq 3^{n} J_{1}$, where $J_{1}:=\sup _{P} \phi-\sup _{\frac{1}{2} P} \phi$.

Using (3.1) repeatedly we get

Lemma 3.0.5 $I_{2} \leq J_{2}$, where $J_{2}:=f(0)-f(-1 / 2, \ldots,-1 / 2)$ with

$$
f(t):=\phi_{\left\{z:\left|z_{j}-\hat{z}_{j}\right|=e^{t_{j}} r_{j}, 1 \leq j \leq n\right\}} \cdot
$$

Since both $\sup _{P} \phi$ and $\phi_{\partial P}$ are continuous in $\hat{z}_{j}$ and convex increasing with respect to $\log r_{j}$ for all $j$, it follows from Lemma 3.0.3 (through a similar argument as the onedimensional case) that

$$
\sup _{\left.P \in \mathcal{P}_{N}\right|_{\Omega_{0}}}\left(J_{1}+J_{2}\right)<\infty,
$$

for every open set $\Omega_{0} \subset \subset \Omega$, which finishes the proof of the first part of Theorem 1.1.

\subsection{A counterexample}

For the second part of Theorem 1.1, we need to construct a counterexample. For the sake of simplicity, we only consider the case $n=2$. It suffices to verify the following

Theorem 3.1 Set $\phi(z, w):=-\sqrt{(\log |z|+\log |w|) \log |w|}, z, w \in \mathbb{D}$. Then we have $\phi \in$ PSH $\left(\mathbb{D}^{2}\right)$, while

$$
\sup _{0<r_{1}, r_{2}<1} \frac{1}{\left|\mathbb{D}_{r}^{2}\right|} \int_{\mathbb{D}_{r}^{2}}\left|\phi-\phi_{\mathbb{D}_{r}^{2}}\right|=\infty
$$

where

$$
\mathbb{D}_{r}^{2}:=\left\{(z, w) \in \mathbb{C}^{2}:|z|<r_{1},|w|<r_{2}\right\}
$$

The following lemma shows that Fact 1, 2 is no more true for general bidiscs.

Lemma 3.1.1 $f(x, y):=-\sqrt{(x+y) y}$ is convex on $(-\infty, 0)^{2}$ and increasing in each variable; moreover,

$$
\sup _{\{x, y \leq-1\}}[f(x, y)-f(x-1, y-1)]=\infty .
$$

Proof The first conclusion follows by a straightforward calculation. For (3.2) it suffices to note that

$$
f(x,-1)-f(x-1,-2)=\frac{5-x}{\sqrt{6-2 x}+\sqrt{1-x}} \rightarrow \infty
$$

as $x \rightarrow-\infty$. The proof is complete.

Let us first verify that $\phi \notin B U O_{\mathrm{loc}}\left(\mathbb{D}^{2}, \mathcal{P}\right)$.

Lemma 3.1.2 $\sup _{0<r_{1}, r_{2}<1} \sup _{\mathbb{D}_{r}^{2}}\left(\phi-\phi_{\mathbb{D}_{r}^{2}}\right)=\infty$.

Proof With $x=\log r_{1}$ and $y=\log r_{2}$, we get

$$
\sup _{\mathbb{D}_{r}^{2}}\left(\phi-\phi_{\mathbb{D}_{r}^{2}}\right)=f(x, y)-\int_{-\infty}^{0} \int_{-\infty}^{0} f(x+t, y+s) d e^{2 t} d e^{2 s}=: I(x, y) .
$$


Integrate by parts with respect to $t$ and $s$ successively, we may write

$$
I(x, y)=I_{1}+I_{2},
$$

where

$$
I_{1}=\int_{-\infty}^{0} \frac{x+2 y+2 s}{-4 f(x+t, y+s)} d e^{2 s}
$$

and

$$
I_{2}=\int_{(-\infty, 0)^{2}} \frac{y+s}{-4 f(x+t, y+s)} d e^{2 t} d e^{2 s} .
$$

Obviously, $I_{2}(x,-1)$ is bounded on $(-\infty, 0]$, but $I_{1}(x,-1) \rightarrow \infty$ as $x \rightarrow-\infty$, from which the assertion immediately follows.

Proof of Theorem 3.1 By Lemma 3.0.2 we have (still with $x=\log r_{1}, y=\log r_{2}$ )

$$
\begin{aligned}
\phi_{\mathbb{D}_{r}^{2}} & =\int_{-\infty}^{0} \int_{-\infty}^{0} f(x+t, y+s) d e^{2 t} d e^{2 s} \\
& \geq f(x-1 / 2, y-1 / 2)=\sup _{\mathbb{D}_{e^{-1 / 2 r}}^{2}} \phi,
\end{aligned}
$$

which yields

$$
\begin{aligned}
\frac{1}{\left|\mathbb{D}_{r}^{2}\right|} \int_{\mathbb{D}_{r}^{2}} \mid \phi-\phi_{\mathbb{D}_{r}^{2} \mid} & \geq \frac{1}{\left|\mathbb{D}_{r}^{2}\right|} \int_{\mathbb{D}_{e^{-1 / 2}}^{2}}\left(\sup _{\mathbb{D}_{e^{-1 / 2}}^{2}} \phi-\phi\right) \\
& =e^{-2}\left(\sup _{\mathbb{D}_{e^{-1 / 2}}^{2}} \phi-\phi_{\mathbb{D}_{e}^{2}}\right) .
\end{aligned}
$$

By a similar argument as Lemma 3.1.2, we conclude the proof of Theorem 3.1.

\subsection{Lelong class}

In this section we shall prove the third part of Theorem 1.1. The key ingredient is the following counterpart of Lemma 3.0.3.

Lemma 3.1.3 Let $g(t)=g\left(t_{1}, \ldots, t_{n}\right)$ be a convex function on $\mathbb{R}^{n}$ which is increasing in each variable. Assume that

$$
g(t) \leq \max _{1 \leq j \leq n}\left\{\log \left(1+e^{t_{j}}\right)\right\}, \quad \forall t \in \mathbb{R}^{n} .
$$

Then for every $M>0$ we have

$$
\sup _{t \in \mathbb{R}^{n}}[g(t)-g(t-M)] \leq M,
$$

where $t-M:=\left(t_{1}-M, \ldots, t_{n}-M\right)$.

Proof For fixed $t$, we consider the following convex increasing function

$$
f(s):=g\left(t_{1}+s, \ldots, t_{n}+s\right)
$$

on $\mathbb{R}$. Convexity of $f$ gives

$$
\frac{f(0)-f(-M)}{M} \leq \lim _{s \rightarrow \infty} \frac{f(s)-f(0)}{s} .
$$


By the assumption, we have

$$
f(0) \leq f(s) \leq \max _{1 \leq j \leq n}\left\{\log \left(1+e^{t_{j}} e^{s}\right)\right\}
$$

for every $s \geq 0$, so that

$$
\lim _{s \rightarrow \infty} \frac{f(s)-f(0)}{s} \leq 1 .
$$

The proof is complete.

Proof of the third part of Theorem 1.1 Again for any polydisc

$$
P:=\left\{z \in \mathbb{C}^{n}:\left|z_{j}-\hat{z}_{j}\right|<r_{j}, 1 \leq j \leq n\right\},
$$

we may write

$$
U O_{P}(\phi)=\sup _{P} \phi-\phi_{P}=I_{1}+I_{2},
$$

where

$$
I_{1}:=\sup _{P} \phi-\phi_{\partial P}, \quad I_{2}:=\phi_{\partial P}-\phi_{P}
$$

By Lemma 3.0.4 we have

$$
I_{1} \leq 3^{n}\left(\sup _{P} \phi-\sup _{\frac{1}{2} P} \phi\right)
$$

Put

$$
P_{t}:=\left\{z \in \mathbb{C}^{n}:\left|z_{j}-\hat{z}_{j}\right|<e^{t_{j}} r_{j}, 1 \leq j \leq n\right\}
$$

and $f_{1}(t):=\sup _{P_{t}} \phi$. Since $\phi \in \mathcal{L}$, we know that for some constant $c_{1} \gg 1$ the function $f_{1}-c_{1}$ satisfies the assumption in Lemma 3.1.3, so that

$$
\sup _{P} \phi-\sup _{\frac{1}{2} P} \phi=f_{1}(0)-f_{1}(-\log 2) \leq \log 2,
$$

which in turn implies

$$
I_{1} \leq 3^{n} \log 2 .
$$

Moreover, we infer from Lemma 3.0.5 that

$$
I_{2} \leq f(0)-f(-1 / 2, \ldots,-1 / 2), \quad f(t):=\phi_{\partial P_{t}} .
$$

Applying Lemma 3.1.3 in a similar way as above, we have

$$
I_{2} \leq 1 / 2 \text {. }
$$

Thus

$$
U O_{P}(\phi) \leq 3^{n} \log 2+1 / 2<3^{n},
$$

which finishes the proof. 


\section{Proof of Theorem 1.2}

The starting point is the following

Definition 4.0.1 ( $\gamma$-constant) We shall define the constant $\gamma$ as the BUO norm of $\log |z|$ on $\mathbb{C}$ with respect to all line segments. More precisely,

$$
\gamma:=\sup _{a \neq b \in \mathbb{C}} U O_{[a, b]}(\log |z|),
$$

where $[a, b]$ denotes the line segment connecting $a$ and $b$, and the upper oscillation is defined by

$$
U O_{[a, b]}(\log |z|):=\left(\sup _{0 \leq t \leq 1} \log |a(1-t)+b t|\right)-\int_{0}^{1} \log |a(1-t)+b t| d t .
$$

The key step is to show the following

Lemma 4.0.4 $1<\gamma<2$ is determined by

$$
\gamma+\log (\gamma-1)=0 .
$$

Proof For each pair $a, b \in \mathbb{C}$, we shall compute

$$
U O_{[a, b]}(\log |z|)=\sup _{[a, b]} \log |z|-(\log |z|)_{[a, b]} .
$$

Since $\log |z|$ is $S^{1}$-invariant, by a rotation of $z$, we may assume that

$$
b \in \mathbb{R}, \quad b>|a| .
$$

Thus

$$
\sup _{[a, b]} \log |z|=\log b
$$

is independent of $a$. Since

$$
\begin{aligned}
(\log |z|)_{[a, b]} & =\int_{0}^{1} \log |a(1-t)+b t| d t \\
& \geq \int_{0}^{1} \log |\operatorname{Re} a \cdot(1-t)+b t| d t \\
& =(\log |z|)_{[\operatorname{Re} a, b]}
\end{aligned}
$$

with equality holds if and only if $a \in \mathbb{R}$. Thus it suffices to verify (4.1) for

$$
a, b \in \mathbb{R}, \quad|a|<b .
$$

Consider $\log |z|-\log b$ instead of $\log |z|$, one may further assume that

$$
b=1, \quad-1<a<1,
$$

which implies

$$
U O_{[a, 1]}(\log |z|)=\log 1-(\log |z|)_{[a, 1]}=-(\log |z|)_{[a, 1]} .
$$

We divide into two cases. (i) $0 \leq a<1$. Then we have

$$
-(\log |z|)_{[a, 1]}=\frac{-1}{1-a} \int_{a}^{1} \log x d x=\frac{a \log a}{1-a}+1 \leq 1 .
$$


(ii) $-1<a<0$. Then we have

$$
-(\log |z|)_{[a, 1]}=\frac{-1}{1-a} \int_{a}^{1} \log |x| d x=\frac{a \log (-a)}{1-a}+1>1 .
$$

Thus

$$
\gamma=\sup _{-1<a<0} \frac{a \log (-a)}{1-a}+1 .
$$

It suffices to verify that $\gamma$ satisfies (4.1). To see this, put

$$
t^{-1}:=1-a \in(1,2)
$$

and write

$$
\frac{a \log (-a)}{1-a}=(1-t) \log t-(1-t) \log (1-t)=: f(t)
$$

Since

$$
f^{\prime}(t)=t^{-1}-\log t+\log (1-t),
$$

it follows that $f^{\prime}(t)=0$ if and only if

$$
t^{-1}=\log \frac{1}{t^{-1}-1},
$$

i.e.,

$$
1-a+\log (-a)=0
$$

Thus we have

$$
\gamma=\sup _{-1<a<0} \frac{a \log (-a)}{1-a}+1=\frac{a_{0} \log \left(-a_{0}\right)}{1-a_{0}}+1,
$$

where $a_{0}$ is determined by

$$
1-a_{0}+\log \left(-a_{0}\right)=0
$$

which gives

$$
\gamma=1-a_{0} \in(1,2) .
$$

It is clear that (4.2) is equivalent to (4.1).

Since a translation of a line segment is still a line segment, we know that $\log \left|z-z_{0}\right|$ and $\log |z|$ have the same line segment BUO norm. This fact can be used to estimate the line segment BUO norm of $\log |p|$ for general polynomials $p$. In fact, if we write

$$
p=a_{0}\left(z-a_{1}\right)^{n_{1}} \cdots\left(z-a_{k}\right)^{n_{k}},
$$

then

$$
\sup _{[a, b]} \log |p| \leq \log \left|a_{0}\right|+\sum_{j=1}^{k} n_{j} \sup _{[a, b]} \log \left|z-a_{j}\right|
$$


and

$$
(\log |p|)_{[a, b]}=\log \left|a_{0}\right|+\sum_{j=1}^{k} n_{j}\left(\log \left|z-a_{j}\right|\right)_{[a, b]} .
$$

Thus

$$
\begin{aligned}
U O_{[a, b]}(\log |p|) & :=\sup _{[a, b]} \log |p|-(\log |p|)_{[a, b]} \\
& \leq \sum_{j=1}^{k} n_{j} U O_{[a, b]}\left(\log \left|z-a_{j}\right|\right) .
\end{aligned}
$$

This combined with the fact $U O_{[a, b]}\left(\log \left|z-a_{j}\right|\right) \leq \gamma$ gives

$$
U O_{[a, b]}(\log |p|) \leq \gamma \cdot \operatorname{deg} p .
$$

for all polynomials $p$ and all $a, b \in \mathbb{C}$.

Now we may conclude the proof of Theorem 1.2 as follows. Since $A$ is compact, we may choose $z_{0} \in A$ such that

$$
\left|p\left(z_{0}\right)\right|=\sup _{z \in A}|p(z)| .
$$

For every ray (half line), say $L$, starting from $z_{0}$, we see that $A \cap L$ is a line segment in view of convexity of $A$. Let $L_{\mathbb{C}}$ be the complex line containing $L$. Apply (4.3) to $\left.p\right|_{L_{\mathbb{C}}}$, we have

$$
U O_{A \cap L}(\log |p|)=U O_{A \cap L}\left(\log |p|_{L_{\mathbb{C}}} \mid\right) \leq\left.\gamma \operatorname{deg} p\right|_{L_{\mathbb{C}}} \leq \gamma \operatorname{deg} p
$$

which gives

$$
U O_{A}(\log |p|) \leq \gamma \operatorname{deg} p
$$

since $U O_{A}(\log |p|)$ is a certain average of $U O_{A \cap L}(\log |p|)$ for all $L$ starting from $z_{0}$ : in fact, since $z_{0}$ is a maximum point of $\log |p|$ on $A$ and $L$ contains $z_{0}$, we always have

$$
\sup _{A \cap L} \log |p|=\log \left|p\left(z_{0}\right)\right|,
$$

together with (4.3) it gives

$$
\gamma \cdot \operatorname{deg} p \geq U O_{A \cap L}(\log |p|)=\log \left|p\left(z_{0}\right)\right|-\frac{1}{|A \cap L|} \int_{A \cap L} \log |p| .
$$

Thus

$$
\begin{aligned}
\int_{A} \log |p| & =\int_{S_{2 n-1}} \int_{A \cap L} \log |p| d \mu(L) \\
& \geq\left(\log \left|p\left(z_{0}\right)\right|-\gamma \cdot \operatorname{deg} p\right) \int_{S_{2 n-1}}|A \cap L| d \mu(L) \\
& =\left(\log \left|p\left(z_{0}\right)\right|-\gamma \cdot \operatorname{deg} p\right)|A|,
\end{aligned}
$$

where $d \mu$ is a certain measure on the unit sphere $S_{2 n-1}$ and we identify the set of rays $L$ starting from $z_{0}$ with $S_{2 n-1}$. Notice that the above inequality gives

$$
\gamma \cdot \operatorname{deg} p \geq \log \left|p\left(z_{0}\right)\right|-\frac{1}{|A|} \int_{A} \log |p|=U O_{A}(\log |p|),
$$

from which the assertion immediately follows. 


\section{Proof of Theorem 1.3}

The starting point is the following

Proposition 5.0.1 (John-Nirenberg inequality) Suppose $\phi \in P S H(\Omega)$ and $\Omega_{0} \subset \subset \Omega$ is open. For each $a=\left(a_{1}, \ldots, a_{n}\right)$ with all $a_{j}>0$ there exists $\varepsilon_{0}=\varepsilon\left(a, \phi, \Omega_{0}, \Omega\right)>0$ such that

$$
\sup _{P_{r} a(\hat{z}) \subset \Omega_{0}} \frac{1}{\left|P_{r^{a}}(\hat{z})\right|} \int_{P_{r^{a}}(\hat{z})} e^{-\varepsilon\left(\phi-\sup _{P_{r} a(\hat{z})} \phi\right)}<\infty,
$$

for every $\varepsilon \leq \varepsilon_{0}$. Here

$$
P_{r^{a}}(\hat{z})=\left\{z \in \mathbb{C}^{n}:\left|z_{j}-\hat{z}_{j}\right| \leq r^{a_{j}}\right\} .
$$

Although the argument is fairly standard, we will provide a proof in Appendix, because the result cannot be found in literature explicitly.

Lemma 5.0.5 Let $\psi$ be a psh function on $\Omega$ which satisfies $\sup _{\Omega} \psi<\infty$ and $\int_{\Omega} e^{-\psi}<\infty$. Suppose $\Omega$ is circular, i.e., $\zeta z \in \Omega$ for every $\zeta \in \mathbb{C},|\zeta| \leq 1$, and $z \in \Omega$. Then

$$
\left(\frac{1}{|\Omega|} \int_{\Omega} e^{-\left(\psi-\sup _{\Omega} \psi\right)}\right)^{-1} \leq K_{\psi, \Omega}(0) \cdot|\Omega| \cdot e^{-\sup _{\Omega} \psi} \leq 1 .
$$

Proof The extremal property of the Bergman kernel implies that

$$
K_{\psi, \Omega}(0) \geq \frac{1}{\int_{\mathbb{D}_{r}} e^{-\psi}}
$$

and the first inequality in (5.1) holds. On the other hand, as $\Omega$ is circular, it is easy to verify that

$$
f(0)=\frac{1}{|\Omega|} \int_{\Omega} f
$$

for all $f \in \mathcal{O}(\Omega)$. Thus we have

$$
|f(0)|^{2}=\left|\frac{1}{|\Omega|} \int_{\Omega} f\right|^{2} \leq \frac{1}{|\Omega|} \int_{\Omega}|f|^{2} e^{-\psi} \cdot e^{\sup _{\Omega} \psi},
$$

so that the second inequality in (5.1) also holds.

Proof of Theorem 1.3 Since

$$
\int_{\mathbb{D}^{n}}|f|^{2} e^{-\varepsilon \phi^{t}}=\left|t_{1} \cdots t_{n}\right|^{-2} \int_{\mathbb{D}_{t}^{n}}|f|^{2} e^{-\varepsilon \phi}, \quad \forall f \in \mathcal{O}(\Omega),
$$

it follows that

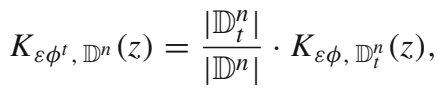

where

$$
\mathbb{D}_{t}^{n}:=\left\{z \in \mathbb{C}^{n}:\left|z_{j}\right|<\left|t_{j}\right|, 1 \leq j \leq n\right\} .
$$

Thus we have

$$
F(\varepsilon \phi)(t, 0)=\log \left(\left|\mathbb{D}_{t}^{n}\right| \cdot K_{\varepsilon \phi}, \mathbb{D}_{t}^{n}(0)\right)-n \log \pi .
$$


This combined with Lemma 5.0.5 gives

$$
-\log \left(\frac{1}{\left|\mathbb{D}_{t}^{n}\right|} \int_{\mathbb{D}_{t}^{n}} e^{-\varepsilon\left(\phi-\sup _{\mathbb{D}_{t}^{n}} \phi\right)}\right)-n \log \pi \leq F(\varepsilon \phi)(t, 0)-\varepsilon \sup _{\mathbb{D}_{t}^{n}} \phi \leq-n \log \pi .
$$

By Proposition 5.0.1, we conclude the proof.

\section{Proof of Theorem 1.4}

Recall that

$$
\phi^{t}(z):=\phi\left(t_{1} z_{1}, \ldots, t_{n} z_{n}\right) .
$$

By Proposition 2.2 in [3], we have

$$
\frac{\partial}{\partial t_{j}} K_{\phi^{t}, \mathbb{D}^{n}}(0)=\int_{\mathbb{D}^{n}} \frac{\partial \phi^{t}}{\partial t_{j}}\left|K_{\phi^{t}, \mathbb{D}^{n}}(z, 0)\right|^{2} e^{-\phi^{t}},
$$

where $K_{\phi^{t}, \mathbb{D}^{n}}(z, 0)$ satisfies the following reproducing property

$$
f(0)=\int_{\mathbb{D}^{n}} f(z) \overline{K_{\phi^{t}, \mathbb{D}^{n}}(z, 0)} e^{-\phi^{t}}
$$

for all $L^{2}$ holomorphic functions $f$ on $\mathbb{D}^{n}$. In particular, if $f=z K_{\phi^{t}, \mathbb{D}^{n}}(z, 0)$ then

$$
0=\int_{\mathbb{D}^{n}} z \cdot\left|K_{\phi^{t}, \mathbb{D}^{n}}(z, 0)\right|^{2} e^{-\phi^{t}}
$$

and since $\left.\frac{\partial \phi^{t}}{\partial t_{j}}\right|_{t=0}=z_{j} \phi_{z_{j}}(0)$, we get

$$
\left.\int_{\mathbb{D}^{n}} \frac{\partial \phi^{t}}{\partial t_{j}}\right|_{t=0}\left|K_{\phi^{t}, \mathbb{D}^{n}}(z, 0)\right|^{2} e^{-\phi^{t}}=0
$$

for all $t \in \mathbb{D}^{n}$. Thus we may write (6.1) as

$$
\frac{\partial}{\partial t_{j}} K_{\phi^{t}, \mathbb{D}^{n}}(0)=\int_{\mathbb{D}^{n}}\left(\frac{\partial \phi^{t}}{\partial t_{j}}-\left.\frac{\partial \phi^{t}}{\partial t_{j}}\right|_{t=0}\right)\left|K_{\phi^{t}, \mathbb{D}^{n}}(z, 0)\right|^{2} e^{-\phi^{t}}
$$

In particular,

$$
\left.\frac{\partial}{\partial t_{j}} K_{\phi^{t}, \mathbb{D}^{n}}(0)\right|_{t=0}=0
$$

Thus we can further write (6.1) as

$$
\frac{\partial}{\partial t_{j}} K_{\phi^{t}, \mathbb{D}^{n}}(0)-\left.\frac{\partial}{\partial t_{j}} K_{\phi^{t}, \mathbb{D}^{n}}(0)\right|_{t=0}=\int_{\mathbb{D}^{n}}\left(\frac{\partial \phi^{t}}{\partial t_{j}}-\left.\frac{\partial \phi^{t}}{\partial t_{j}}\right|_{t=0}\right)\left|K_{\phi^{t}, \mathbb{D}^{n}}(z, 0)\right|^{2} e^{-\phi^{t}},
$$

which implies

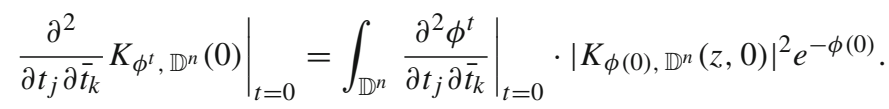

Since

$$
K_{\phi(0), \mathbb{D}^{n}}(z, 0)=\frac{e^{\phi(0)}}{\pi^{n}}
$$


and

$$
\left.\frac{\partial^{2} \phi^{t}}{\partial t_{j} \partial \bar{t}_{k}}\right|_{t=0}=z_{j} \bar{z}_{k} \phi_{z_{j} \bar{z}_{k}}(0)
$$

we get

$$
\left.\frac{\partial^{2}}{\partial t_{j} \partial \bar{t}_{k}} K_{\phi^{t}, \mathbb{D}^{n}}(0)\right|_{t=0}=\frac{e^{\phi(0)} \phi_{z_{j} \bar{z}_{k}}(0)}{\pi^{2 n}} \int_{\mathbb{D}^{n}} z_{j} \bar{z}_{k} .
$$

Notice that

$$
\int_{\mathbb{D}^{n}} z_{j} \bar{z}_{k}= \begin{cases}\pi^{n} / 2 & \text { if } j=k \\ 0 & \text { if } j \neq k\end{cases}
$$

and

$$
\left.\frac{\partial^{2}}{\partial t_{j} \partial \bar{t}_{k}} K_{\phi^{t}, \mathbb{D}^{n}}(0)\right|_{t=0}=\left.K_{\phi^{t}, \mathbb{D}^{n}}(0) \cdot \frac{\partial^{2}}{\partial t_{j} \partial \bar{t}_{k}} \log K_{\phi^{t}, \mathbb{D}^{n}(0)}\right|_{t=0},
$$

our assertion follows.

Acknowledgements Open Access funding provided by NTNU Norwegian University of Science and Technology (incl St. Olavs Hospital - Trondheim University Hospital) The authors would like to thank Ahmed Zeriahi for bringing their attention to the reference [1]. The second author would like to thank Bo Berndtsson for numerous useful discussions about the topics of this paper.

Open Access This article is licensed under a Creative Commons Attribution 4.0 International License, which permits use, sharing, adaptation, distribution and reproduction in any medium or format, as long as you give appropriate credit to the original author(s) and the source, provide a link to the Creative Commons licence, and indicate if changes were made. The images or other third party material in this article are included in the article's Creative Commons licence, unless indicated otherwise in a credit line to the material. If material is not included in the article's Creative Commons licence and your intended use is not permitted by statutory regulation or exceeds the permitted use, you will need to obtain permission directly from the copyright holder. To view a copy of this licence, visit http://creativecommons.org/licenses/by/4.0/.

\section{Appendix}

In this section we provide a proof of Proposition 5.0.1. Let us first recall a few basic facts in real-variable theory, by following Stein [17]. A quasi-distance defined on $\mathbb{R}^{m}$ means a nonnegative continuous function $\rho$ on $\mathbb{R}^{m} \times \mathbb{R}^{m}$ for which there exists a constant $c>0$ such that

1. $\rho(x, y)=0$ iff $x=y$;

2. $\rho(x, y) \leq c \rho(y, x)$

3. $\rho(x, y) \leq c(\rho(x, z)+\rho(y, z))$.

Given such a $\rho$, we define "balls"

$$
B(x, r):=\{y: \rho(y, x)<r\}, \quad r>0 .
$$

One can verify that there exists a constant $c_{1}>1$ such that for all $x, y$ and $r$,

$$
B(x, r) \cap B(y, r) \neq \emptyset \Rightarrow B(y, r) \subset B\left(x, c_{1} r\right) .
$$


In the case of Proposition 5.0.1, we define

$$
\rho(z, w)=\max _{k}\left|z_{k}-w_{k}\right|^{1 / a_{k}}, \quad z, w \in \mathbb{C}^{n} .
$$

It is easy to verify that $\rho$ is a quasi-distance on $\mathbb{C}^{n}$ and

$$
B(\hat{z}, r)=P_{r^{a}}(\hat{z}), \quad \hat{z} \in \mathbb{C}^{n}, r>0 .
$$

Besides (7.1), the following properties also hold for $B(\hat{z}, r)$ :

$$
\begin{aligned}
& \left|B\left(\hat{z}, c_{1} r\right)\right| \leq c_{1}^{2 \sum_{k} a_{k}} \cdot|B(\hat{z}, r)|=: c_{2}|B(\hat{z}, r)| ; \\
& \bigcap_{r} \bar{B}(\hat{z}, r)=\{\hat{z}\} \quad \text { and } \bigcup_{r} B(\hat{z}, r)=\mathbb{C}^{n} ;
\end{aligned}
$$

For each open set $U$ and each $r>0$, the function $\hat{z} \mapsto|B(\hat{z}, r) \cap U|$ is continuous.

Fix a pair of positive constants $c^{*}$ and $c^{* *}$ with $1<c^{*}<c^{* *}$. For $B=B(\hat{z}, r)$ we define $B^{*}=B\left(\hat{z}, c^{*} r\right)$ and $B^{* *}=B\left(\hat{z}, c^{* *} r\right)$. Then we have

Lemma 7.0.6 (cf. [17, p. 15-16]) Choose $c^{*}=4 c_{1}^{2}$ and $c^{* *}=16 c_{1}^{2}$. Given a closed nonempty set $F \subset \mathbb{C}^{n}$, there exists a collection of balls $\left\{B_{k}\right\}$ such that

(1) The $B_{k}$ are pairwise disjoint;

(2) $\bigcup_{k} B_{k}^{*}=F^{c}:=\mathbb{C}^{n} \backslash F$;

(3) $B_{k}^{* *} \cap F \neq \emptyset$ for each $k$.

Proposition 7.0.2 (Calderón-Zygmund decomposition) Let $B_{0}$ be a ball in $\mathbb{C}^{n}$ and $f \in$ $L^{1}\left(B_{0}\right)$. There is a constant $c=c\left(c_{1}, c_{2}\right)>0$ such that given a positive number $\alpha$, there exists a sequences of balls $\left\{B_{k}^{*}\right\}$ in $B_{0}$ such that

(1) $|f(z)| \leq \alpha$, for a.e. $z \in B_{0} \backslash \bigcup_{k} B_{k}^{*}$;

(2) $\int_{B_{k}^{*}}|f| \leq c \alpha\left|B_{k}^{*}\right|$, for each $k$;

(3) $\sum_{k}\left|B_{k}^{*}\right| \leq \frac{c}{\alpha} \int_{B_{0}}|f|$.

Proof We extend $f$ to an integrable function on $\mathbb{C}^{n}$ by setting $f=0$ outside $B_{0}$. Recall the following two types of Hardy-Littlewood maximal functions:

$$
\begin{aligned}
& M f(z):=\sup _{r>0} \frac{1}{|B(z, r)|} \int_{B(z, r)}|f|, \\
& \tilde{M} f(z):=\sup _{z \in B} \frac{1}{|B|} \int_{B}|f|
\end{aligned}
$$

where the supremum is taken over all balls $B$ containing $z$. The relationship between $M f$ and $\tilde{M} f$ is as follows:

$$
M f \leq \tilde{M} f \leq c_{2} M f
$$

Notice that

$$
E_{\alpha}:=\left\{z \in B_{0}: \tilde{M} f(z)>\alpha\right\}
$$

is an open set since $\tilde{M} f$ is lower semicontinuous, and

$$
\left|E_{\alpha}\right| \leq \frac{c}{\alpha} \int_{\mathbb{C}^{n}}|f|=\frac{c}{\alpha} \int_{B_{0}}|f|
$$


in view of (7.5) and [17, p. 13, Theorem 1]. Here and in what follows $c$ will denote a generic positive constant depending only on $c_{1}, c_{2}$. With $F:=\mathbb{C}^{n} \backslash E_{\alpha}$ we choose balls $\left\{B_{k}\right\},\left\{B_{k}^{*}\right\}$ and $\left\{B_{k}^{* *}\right\}$ according to Lemma 7.0.6. Then we have

$$
\sum_{k}\left|B_{k}^{*}\right| \leq c \sum_{k}\left|B_{k}\right| \leq c\left|E_{\alpha}\right| \leq \frac{c}{\alpha} \int_{B_{0}}|f| .
$$

Since $B_{k}^{* *} \cap F \neq \emptyset$ for each $k$, we have

$$
\int_{B_{k}^{*}}|f| \leq \int_{B_{k}^{* *}}|f| \leq \alpha\left|B_{k}^{* *}\right| \leq c \alpha\left|B_{k}^{*}\right| .
$$

Finally, by (7.5) and [17, p. 13, Corollary], we know that $|f(z)| \leq \widetilde{M} f(z)$ for a.e. $z$, from which (1) immediately follows.

Proof of Proposition 5.0.1 By Theorem 1.1, we know that

$$
M:=\sup _{B(\hat{z}, r) \subset \Omega_{0}} \frac{1}{|B(\hat{z}, r)|} \int_{B(\hat{z}, r)}\left|\phi-\phi_{B(\hat{z}, r)}\right|<\infty .
$$

Assume without loss of generality $M=1$. Fix a ball $B_{0} \subset \Omega_{0}$. It suffices to show

$$
\left|\left\{z \in B_{0}:\left|\phi-\phi_{B_{0}}\right|>t\right\}\right| \leq \text { const } \cdot e^{-\varepsilon t}\left|B_{0}\right|, \quad t>0,
$$

for certain $\varepsilon \ll 1$. With $c$ as Proposition 7.0.2 we choose

$$
\alpha>c>1 \geq \frac{1}{\left|B_{0}\right|} \int_{B_{0}}\left|\phi-\phi_{B_{0}}\right| .
$$

Applying Proposition 7.0.2 with $f=\left|\phi-\phi_{B_{0}}\right|$, we have a sequence of balls $\left\{B_{k}^{(1)}\right\}$ in $B_{0}$ such that

$$
\begin{aligned}
& \left|\phi(z)-\phi_{B_{0}}\right| \leq \alpha \quad \text { a.e. } \quad z \in B_{0} \backslash \bigcup_{k} B_{k}^{(1)}, \\
& \sum_{k}\left|B_{k}^{(1)}\right| \leq \frac{c}{\alpha} \int_{B_{0}}\left|\phi-\phi_{B_{0}}\right| \leq \frac{c}{\alpha}\left|B_{0}\right|,
\end{aligned}
$$

and

$$
\left|\phi_{B_{k}^{(1)}}-\phi_{B_{0}}\right| \leq \frac{1}{\left|B_{k}^{(1)}\right|} \int_{B_{k}^{(1)}}\left|\phi-\phi_{B_{0}}\right| \leq c \alpha .
$$

Applying Proposition 7.0.2 with $f=\left|\phi-\phi_{B_{k}^{(1)}}\right|$ for each $k$, we obtain a sequence of balls $\left\{B_{k}^{(2)}\right\}$ in $\bigcup_{k} B_{k}^{(1)}$ such that

$$
\sum_{k}\left|B_{k}^{(2)}\right| \leq \frac{c}{\alpha} \sum_{k} \int_{B_{k}^{(1)}}\left|\phi-\phi_{B_{k}^{(1)}}\right| \leq \frac{c}{\alpha} \sum_{k}\left|B_{k}^{(1)}\right| \leq \frac{c^{2}}{\alpha^{2}}\left|B_{0}\right|
$$

and

$$
\left|\phi(z)-\phi_{B_{k}^{(1)}}\right| \leq \alpha \quad \text { a.e. } \quad z \in B_{k}^{(1)} \backslash \bigcup_{k} B_{k}^{(2)},
$$

which in turn implies

$$
\left|\phi(z)-\phi_{B_{0}}\right| \leq 2 \cdot c \alpha \quad \text { a.e. } \quad z \in B_{0} \backslash \bigcup_{k} B_{k}^{(2)} .
$$


Continue this process. For each $j$ there exists a sequence of balls $\left\{B_{k}^{(j)}\right\}$ in $\bigcup_{k} B_{k}^{(j-1)}$ such that

$$
\begin{aligned}
& \sum_{k}\left|B_{k}^{(j)}\right| \leq \frac{c^{j}}{\alpha^{j}}\left|B_{0}\right|, \\
& \left|\phi(z)-\phi_{B_{0}}\right| \leq j \cdot c \alpha \quad \text { a.e. } z \in B_{0} \backslash \bigcup_{k} B_{k}^{(j)} .
\end{aligned}
$$

Thus

$$
\left|\left\{z \in B_{0}:\left|\phi-\phi_{B_{0}}\right|>j \cdot c \alpha\right\}\right| \leq \sum_{k}\left|B_{k}^{(j)}\right| \leq \frac{c^{j}}{\alpha^{j}}\left|B_{0}\right| .
$$

For any $t$ there exists an integer $j$ such that $t \in[j \cdot c \alpha,(j+1) \cdot c \alpha)$. It follows that

$$
(c / \alpha)^{j}=(\alpha / c) e^{-(j+1) \log \alpha / c} \leq(\alpha / c) e^{-\frac{\log \alpha / c}{c \alpha} t},
$$

from which (7.6) immediately follows. Now we have

$$
\frac{1}{\left|B_{0}\right|} \int_{B_{0}} e^{\varepsilon\left|\phi-\phi_{B_{0}}\right|}=\frac{1}{\left|B_{0}\right|} \int_{0}^{\infty} e^{\varepsilon t}\left|\left\{\left|\phi-\phi_{B_{0}}\right|>t\right\}\right|+\frac{\left|\left\{\phi=\phi_{B_{0}}\right\}\right|}{\left|B_{0}\right|} \leq \text { const }+1,
$$

which gives

$$
\frac{1}{\left|B_{0}\right|} \int_{B_{0}} e^{-\varepsilon\left(\phi-\sup _{B_{0}} \phi\right)} \leq(\text { const }+1) e^{\varepsilon\left(\sup _{B_{0}} \phi-\phi_{B_{0}}\right)},
$$

By Theorem 1.1, $\sup _{B_{0}}\left\{\sup _{B_{0}} \phi-\phi_{B_{0}}\right\}<\infty$, thus Proposition 5.0.1 follows.

\section{References}

1. Benelkourchi, S., Jennane, B., Zeriahi, A.: Polya's inequalities, global uniform integrability and the size of plurisubharmonic lemniscates. Ark. Mat. 43, 85-112 (2005)

2. Berndtsson, B.: Subharmonicity properties of the Bergman kernel and some other functions associated to pseudoconvex domains. Ann. Inst. Fourier (Grenoble) 56, 1633-1662 (2006)

3. Berndtsson, B.: A Comparison Principle for Bergman Kernels, Analysis Meets Geometry: A Tribute to Mikael Passare. Trends in Mathematics, pp. 121-126 (2017)

4. Berndtsson, B., Lempert, L.: A proof of the Ohsawa-Takegoshi theorem with sharp estimates. J. Math. Soc. Jpn. 68, 1461-1472 (2016)

5. Blocki, Z.: Suita's conjecture and the Ohsawa-Takegoshi extension theorem. Invent. Math. 193, 149-158 (2013)

6. Brudnyi, A.: Local inequalities for plurisubharmonic functions. Ann. Math. 149, 511-533 (1999)

7. Brudnyi, A.: On a BMO-property for subharmonic functions. J. Fourier Anal. Appl. 8, 603-612 (2002)

8. Brudnyi, Y., Ganzburg, M.: On an extremal problem for polynomials of $n$ variables. Math. USSR Izv. 37, 344-355 (1973)

9. Chen, B.Y.: Weighted Bergman kernel, directional Lelong number and John-Nirenberg exponent. J. Geom. Anal. 30, 1271-1292 (2020)

10. Coifman, R.R., Rochberg, R.: Another characterization of BMO. Proc. Am. Math. Soc. 79, 249-254 (1980)

11. Demailly, J.P.: Complex Analytic and Differential Geometry, open book available in his homepage

12. Guan, Q., Zhou, X.: A solution of an $L^{2}$-extension problem with an optimal estimate and applications. Ann. Math. 181, 1139-1208 (2015)

13. John, F., Nirenberg, L.: On functions of bounded mean oscillation. Commun. Pure Appl. Math. 14, 415-426 (1961)

14. Kiselman, C.O.: Attenuating the singularities of plurisubharmonic functions. Ann. Polon. Math. 60, 173197 (1994)

15. Nazarov, F., Sodin, M., Vol'berg, A.: The geometric Kannan-Lovász-Simonovits lemma, dimensionfree estimates for volume of subleval sets of polynomials, and distribution of zeros of random analytic functions. arXiv:math/0108212v2 
16. Osekowski, A.: Sharp estimates for functions of bounded lower oscillation. Bull. Aust. Math. Soc. 87, 68-81 (2013)

17. Stein, E.M.: Harmonic Analysis: Real-Variable Methods, Orthogonality, and Oscillatory Integrals. Princeton University Press, Princeton (1993)

Publisher's Note Springer Nature remains neutral with regard to jurisdictional claims in published maps and institutional affiliations. 